\title{
Human Papillomavirus as an Independent Predictor in Oral Squamous Cell Cancer
}

\author{
Dan Zhao ${ }^{1, a}$, Qin-gan $X u^{1, a}$, Xin-ming Chen ${ }^{1,2}$, Ming-wen Fan ${ }^{1, *}$ \\ ${ }^{1}$ Key Laboratory for Oral Biomedical Engineering of Ministry of Education, Wuhan University, Wuhan, China \\ ${ }^{2}$ Department of Pathology, School \& Hospital of Stomatology, Wuhan University, Wuhan, China
}

\begin{abstract}
Dan Zhao, Qin-gan Xu, Xin-ming Chen, Ming-wen Fan. Human Papillomavirus as an Independent Predictor in Oral Squamous Cell Cancer. International Journal of Oral Science, 1(3): 119-125, 2009

Aim There is an increasing evidence for the role of high risk human papillomavirus (HPV) in the pathogenesis of oral squamous cell carcinoma (OSCC). The purpose of this study is to evaluate the relevance of HPV infection to the survival and prognosis of OSCC.

Methodology Fifty-two patients with OSCC were followed from 4 to 88 months with a median of 50.7 months. HPV DNA was identified in formalin-fixed, paraffin-embedded tumor specimens by nested PCR with MY09/MY11 and $\mathrm{GP5}^{+} / \mathrm{GP}^{+}$primer pairs and the HPV genotype was determined by direct DNA sequencing. Association between the HPV status and risk factors for cancer as well as tumor-host characteristics were analyzed.
\end{abstract}

Survival curves were calculated by the Kaplan-Meier method and analyzed using the log-rank test.

Results HPV was found in $40.4 \%$ of the tumors with HPV 16 accounting for $63.5 \%$, HPV 18 for $30.8 \%$, HPV 6 for $3.9 \%$ and HPV 11 for $1.8 \%$. No infection with more than one HPV genotype was detected. HPV infection was significantly associated with poor histological grade, TNM stage I - II, alcohol usage and no smoking status. Multivariate analysis showed that HPV had an independent prognostic effect on the overall survival after adjusting other confounding factors such as histological grade, TNM stage and tobacco usage. The presence of HPV was significantly correlated with a better survival in patients with OSCC.

Conclusion HPV infection can act as an independent predictor for the survival and prognosis of OSCC.

Keywords human papillomavirus (HPV), oral squamous cell carcinoma (OSCC), survival analysis
Document code: A

CLC number: R373.9

\section{Introduction}

Oral cancer is the sixth most prevalent cancer worldwide, ranking third in developing countries and eighth in developed countries, and China (incl. Hong Kong) is involved in one of the six high-risk areas and countries worldwide (Syrjänen, 2005). In China, the incidence of oral cancer has jumped from $81 / 1,000,000$ to $202 / 1,000,000$ in the past few years, and estimates of mortality in China approach 10,000 annually. Since oral squamous cell carcinomas (OSCCs) are prone to be detected at its late stage, the overall survival rate of such
Received Mar. 23, 2009; Revision accepted Aug. 4, 2009

kind of diseases can only reach about $40 \%-50 \%$.

Alcohol use and cigarette smoking are wellknown risk factors for the development of oral cancer (Decker et al., 1982). In 1983, human papillomavirus (HPV) was first described as a factor involved in the development of OSCC (Syrjänen et al., 1983). From then on, the presence of different types of HPV has been detected in oral cancers, which are mainly HPV16 and HPV 18 with noticeable detection rates from $8 \%$ to $64 \%$ (Kansky, et al., 2003; Lo Muzio et al., 2004; Hansson et al., 2005). 


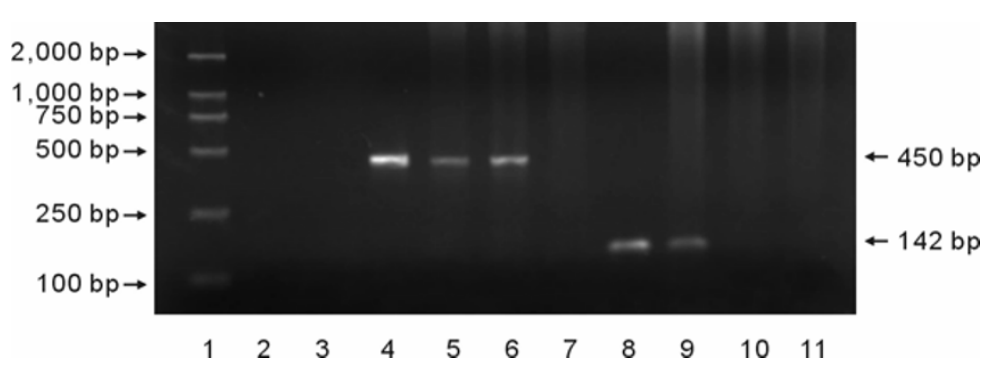

Figure 1 Products of MY-PCR (450 bp) and MY/GP-PCR (142 bp)

Lane 1: DL2000 DNA marker; lane 2: blank control; Lane 3: Wi38 cells (negative control); Lane 4 and 8: Hela cells (positive control); Lane

5, 6 and 9: positive cases; Lane 7, 10 and 11: negative cases.

The presence of HPV DNA in a significant fraction of oral cancer, combined with the evidence that HPV status may affect the clinical outcomes in cervical or anogenital cancer patients (Lombard et al., 1998), led to a number of studies pointing a possible use of HPV diagnosis as an additional aid for survival prognosis in oral cancers (Schwartz et al., 2001; Ritchie et al., 2003), while other groups found no differences in survival by HPV status (Brandwein et al., 1994). However, many of these studies did not use detection methods with high sensitivity, screen for all of the HPV types, nor distinguish between the HPV types, and/or did not control for other confounding factors suspected to influence the survival in cancer patients such as tumor histology, histological grade, TNM stage, alcohol use and cigarette smoking.

OSCC is the most common type of oral cancers which accounts for $80 \%$ of the cases. Because some previous studies have found no change in survival rates by HPV status, while other studies show a more pronounced association, the question remains whether HPV exerts any influence on prognosis and survival in OSCC patients. Thus, the purpose of this study is to investigate the relationship between HPV status and the survival of OSCC patients to remedy deficiencies of previous studies.

\section{Materials and methods}

\section{Subjects}

Fifty-two OSCC patients were obtained from School \& Hospital of Stomatology, Wuhan University from 1999 to 2001. Tumor sites included tongue $(n=21)$, cheek $(n=11)$, gingival $(n=11)$, - 120 - Int J Oral Sci, 1(3): 119-125, 2009 floor of mouth $(n=2)$, palate $(n=5)$, and lip $(n=2)$. Clinical staging according to the 1997 criteria of the International Union Against Cancer (Sobin et al., 1997) was evaluated by reviewing the medical charts and pathologic records. Risk factors such as tobacco and alcohol exposure were investigated by interview. All patients had undergone surgery immediately after their first diagnosis.

\section{Tissue specimen preparation and PCR-based HPV typing}

The $10 \%$ formalin-fixed and paraffin-embedded tumor specimens were serially sectioned $(9 \mathrm{sec}-$ tions) on a microtome. The first and last sections were stained with hematoxylin-eosin to confirm the OSCC diagnosis and to determine the histological grade according to the World Health Organization criteria. The intermediate seven sections of $4 \mu \mathrm{m}$ each were used for DNA extraction with GENTRA DNA Tissue Kit (Gentra Systems Inc., USA). $\beta$-globin PCR was used as an internal control for the equal loading of DNA (Saiki et al., 1985).

When $\beta$-globin was not detected in one sample, additional sections were taken from the same sample and reanalyzed. For HPV DNA detection, nested PCR (using two pairs of primers: MY09/MY11 and $\left.\mathrm{GP}^{+} / \mathrm{GP}^{+}\right)$was performed as described elsewhere (Remmerbach et al., 2004) with smart changes. Three controls were used for each sample described as blank control (containing all reagents except the template DNA), negative control (containing DNA purified cultures of HPV-negative cell line Wi38) and positive control (containing DNA purified cultures of HeLa cells infected with HPV18). All the experiments were duplicated (Figure 1). The PCR assays were performed using 
Gene Amp PCR System 9700 (PE Applied Biosystems, USA), and the PCR products were analyzed on $2 \%$ agarose gels.

The decision of HPV genotyping was based on the DNA sequencing of $\mathrm{MY}^{-}$or $\mathrm{MY} / \mathrm{GP}^{+} \mathrm{PCR}$ fragments. Amplification products were purified by QIAGEN PCR purification and directly sequenced by fluorescent dye-labeled dideoxynucleotides and cycle sequencing methods using the Big Dye Terminator Cycle Sequencing Kit (PE Applied Biosystems, USA). Sequence analysis was performed on the ABI PRISM 310 Genetic Analyser (PE Applied Biosystems, USA). Alignments were obtained from the GenBank on-line BLAST server, and downloaded HPV sequences from the HPV database (http:// hpv-web.1anl.gov). All the working surfaces were treated with sodium hypochlorite before and after operation to prevent contamination.

\section{Statistical Analysis}

Analyses were conducted with Windows SPSS ver. 12 (SPSS Inc., USA). Association between the HPV status, OSCC risk factors, as well as other characteristics of patients were analyzed in cross tables by Pearson's $\chi^{2}$ test and with Fisher's exact test for small groups. Survival was measured by the time from the date of diagnosis to the death of patient. The deaths caused by OSCC were considered as outcomes; while the deaths caused by other factors were censored and the missing values were replaced by series mean method. Univariate analysis with Cox's proportional hazards model was deployed to determine every identified prognostic factor; multivariate analysis with Cox's proportional hazards model was used to explore combined effects. A difference of $P<0.05$ was considered statistically significant.

\section{Results}

\section{HPV infection status analysis}

HPV was detected in $40.4 \%$ (21 of 52 ) of the tumors by nested MY/GP PCR, with HPV16 accounting for $63.5 \%$, HPV 18 for $30.8 \%$, HPV6 for $3.9 \%$ and HPV11 for $1.8 \%$. No other HPV types or coinfection were detected in our study. Table 1 shows the correlation between several clinicopathologic factors and HPV infection. It

Table 1 Correlation between HPV infection and clinicopathologic characteristics for 52 OSCC patients

\begin{tabular}{|c|c|c|c|c|c|}
\hline & \multirow[t]{2}{*}{$n(\%)$} & \multicolumn{3}{|c|}{ HPV infection } & \multirow[t]{2}{*}{ OR $(95 \% \mathrm{Cl})$} \\
\hline & & Positive & Negative & $P$-value & \\
\hline \multicolumn{6}{|l|}{ Age } \\
\hline$=50$ & $17(32.7)$ & 8 & 9 & 0.494 & 1.0 \\
\hline$>50$ & $35(67.3)$ & 13 & 22 & & $0.7(0.2-2.1)$ \\
\hline \multicolumn{6}{|l|}{ Gender } \\
\hline Male & $35(67.3)$ & 15 & 10 & 0.602 & $2.8(0.8-9.9)$ \\
\hline Female & $17(32.7)$ & 6 & 11 & & 1.0 \\
\hline \multicolumn{6}{|l|}{ Histological grade } \\
\hline Poor & $25(48.1)$ & 20 & 5 & $0.000^{*}$ & $104.0(11.2-962.1)$ \\
\hline Well+Moderate & $27(51.9)$ & 1 & 26 & & 1.0 \\
\hline \multicolumn{6}{|l|}{ TNM stage } \\
\hline I + II & $22(42.3)$ & 17 & 5 & $0.000^{*}$ & $22.1(5.2-94.2)$ \\
\hline III+IV & $30(57.7)$ & 4 & 26 & & 1.0 \\
\hline \multicolumn{6}{|l|}{ Drinker } \\
\hline No & $31(59.6)$ & 7 & 5 & $0.001^{*}$ & 1.0 \\
\hline Yes & $21(40.4)$ & 14 & 26 & & $0.4(0.1-1.4)$ \\
\hline \multicolumn{6}{|l|}{ Smoker } \\
\hline No & $21(40.4)$ & 18 & 3 & $0.000^{*}$ & $56.0(10.2-308.5)$ \\
\hline Yes & $31(59.6)$ & 3 & 28 & & 1.0 \\
\hline
\end{tabular}

$* P<0.05$ by $\chi^{2}$ test. 
suggested that HPV infection was significantly correlated with poor histological grade, TNM stage I - II, alcohol usage and no smoking status. However, no statistically significant correlation was found between patients' age or gender and the HPV infection.

\section{Survival analysis}

The follow-up period ranged from 4 to 88 months with a median of 50.7 months. Among the 52 patients, 48 patients $(92.3 \%)$ lived for at least 5 years. Twenty-five patients (48.1\%) died of OSCC, 1 patient (1.9\%) died of other reasons than OSCC, 24 patients $(46.2 \%)$ were known to be alive and 2 patients $(3.8 \%)$ were lost at the end of the followup period. Survival curves were calculated by the Kaplan-Meier method and analyzed using the log-rank test for a total of 52 cases (Figure 2). HPV-positive patients showed better overall survival outcomes than did HPV-negative patients. Besides HPV status, histological grade, TNM stage and exposure to tobacco were found to be significantly associated with a worse prognosis by univariate analysis (Cox's proportional hazards model) (Tables 2, 3). This multivariate analysis was used to control for the four prognostic variables mentioned above. All variables had an independent prognostic effect on the OSCC survival except for the exposure to tobacco.

\section{Discussion}

In our study, HPV was found to have an independent prognostic effect on the overall survival of OSCC after adjusting other confounding factors such as histological grade, TNM stage and tobacco usage. The presence of HPV was significantly correlated with a better survival in patients with OSCC excluding other small histological types of oral cancer to reduce confusion. Unlike previous

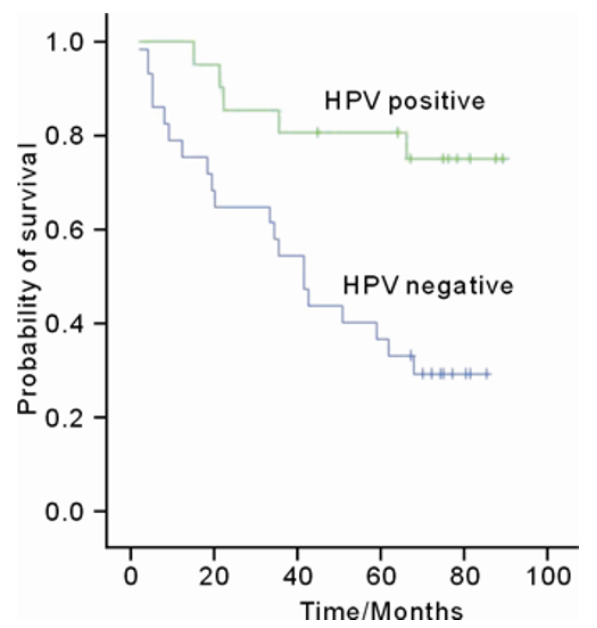

Figure 2 Kaplan-Meier survival curve according to HPV infection $(P=0.0022)$ for 52 OSCC patients

Table 2 Prognostic factors by univariate analysis (Cox's proportional hazards model)

\begin{tabular}{llc}
\hline \multicolumn{1}{c}{ Variables } & \multicolumn{1}{c}{ Hazard ratio* } & $P$-value \\
\hline Age (=50/>50) & $2.027(0.760-5.407)$ & 0.158 \\
Gender (Male/Female) & $1.299(0.542-3.113)$ & 0.557 \\
Histological grade (W+M/P) & $2.360(1.041-5.349)$ & 0.040 \\
TNM stage ( I + II/III+IV) & $25.630(3.490-188.195)$ & 0.001 \\
Drinker (No/Yes) & $0.541(0.233-1.254)$ & 0.152 \\
Smoker (No/Yes) & $2.907(1.159-7.292)$ & 0.023 \\
HPV (Negtive/Positive) & $0.243(0.091-0.651)$ & 0.005 \\
\hline
\end{tabular}

Abbreviations: W, well; M, moderate; P, Poor. ${ }^{* 95 \%}$ confidence interval.

Table 3 Prognostic factors by multiunivariate analysis (Cox's proportional hazards model)

\begin{tabular}{llc}
\hline \multicolumn{1}{c}{ Variables } & \multicolumn{1}{c}{ Hazard ratio* $^{*}$} & $P$-value \\
\hline Histological grade $(\mathrm{W}+\mathrm{M} / \mathrm{P})$ & $0.036(0.004-0.300)$ & 0.002 \\
TNM stage $(\mathrm{I}+\mathrm{II} / \mathrm{III}+\mathrm{IV})$ & $26.383(3.988-174.562)$ & 0.001 \\
Smoker (No/Yes) & $1.618(0.369-7.097)$ & 0.542 \\
HPV (Negtive/Positive) & $0.134(0.018-0.978)$ & 0.048 \\
\hline *95\% confidence interval. & &
\end{tabular}


studies, nested PCR was employed to obtain more sensitive HPV detection and more accurate HPV infection rates, while the HPV genotype was determined by direct DNA sequencing. More than $90 \%$ of the patients had a follow-up until death for more than 5 years. When investigating the relationship between OSCC HPV status and survival, some other confounding factors were adjusted.

This study found that the HPV-positive tumor rate is $40.4 \%$, higher than other reported studies for oral cancer (Yang et al., 2004). One possible explanation could be the sensitive PCR assay that was adopted in our study to overcome this specific problem (Remmerbach et al., 2004). As previously reported, this study confirmed that HPV16 and HPV18 were the two most prevalent HPV types (Syrjänen, 2005). Interestingly, the low-risk HPV types 6 and 11 were also found in our studied cases, although in very small percentages compared to HPV16 and HPV18. This may be caused by the use of MY and GP consensus primers, which allowed for a broad number of HPV genotypes to be detected.

Three risk factors that were highly associated with HPV detection have also been discussed elsewhere (Ritchie et al., 2003; Dahlstrom et al., 2003; Sugiyama et al., 2003; Tachezy et al., 2005; Campisi et al., 2006). Many studies performed by multivariate analysis on HNSCC (Ritchie et al., 2003; Dahlstrom et al., 2003) and one study (Sugiyama et al., 2003) on OSCC have found a correlation between HPV infection and poor histological grade. The same result was obtained in the present study (OR=104.0, 95\% CI: 11.2-962.1). With respect to TNM clinical stage, no link with HPV status was found in most studies on OSCC. These findings were consistent with those reported by Campisi et al., who found TNM stage II and its constituent T2 significantly associated to HPV infection by multivariate analysis and a fuzzy logic (FL) technique (membership functions as input, the ANFIS methodology, and the Sugeno's model of first order) (Campisi et al., 2006). We also located the relationship between the TNM stage I - II and HPV infection (OR=22.1, 95\% CI: 5.2-94.2). Tumors with HPV infection tended to be associated with a decreased exposure to tobacco (OR=56.0, 95\% CI: 10.2-308.5). Our result is in consensus with some other investigations which considered smoking as a protective effect on HPV status in the oral cavity (Ritchie et al., 2003; Tachezy et al., 2005). It has been suggested (Ritchie et al., 2003) that smoking could increase the keratinization of oral mucosal which may make the mucosa more resistant to minor trauma, a proposed mechanism for the necessary infection of the basal layer cells by HPVs.

Interestingly, the presence of HPV was significantly correlated with a better survival of OSCC in the present study, and its prognostic effect on the overall survival was independent after adjusting other confounding factors such as histological grade, TNM stage and tobacco usage. Schwartz et a1. also demonstrated that the presence of HPV16 DNA is independently related to a favorable prognosis in patients with OSCC in a population study of 254 cases (Schwartz et al., 2001). Another large, well-controlled study found that oral cancers containing oncogenic HPV types had $74 \%$ less risk of disease-specific mortality (Gillison et al., 2000). This study provides evidence that the presence of HPV predicted a better survival.

Nevertheless, in a mixed group of head and neck tumors, Hoffmann et al. failed to detect the difference in the overall survival rate between HPV-positive and HPV-negative cancer patients; however, a better survival of HPV-positive patients was demonstrated compared to HPV-negative patients when the same initial nodal status was given (Hoffmann et al., 2005). By investigating 101 HNSCC patients, Pinto et al. also found no significant difference for survival according to the HPV status from HNSCC tumors (Pinto et al., 1999). This study demonstrated evidence, apparently in contrast but not easily comparable since these studies were mainly carried out with HNSCC. Brandwein et al. examined the presence of HPV DNA in the archival specimens of oral carcinomas from 64 patients and found no difference in survival according to HPV status in patients with stage I-II disease; but they observed poorer survival for patients with HPV-positive stage IIIIV disease compared with patients who had HPV-negative stage III-IV disease (Brandwein et al., 1994). However, the population was also characterized by a high degree of occupational exposure to chemical and metallurgic carcinogens. These patients were in addition more likely to be 
HPV-positive (57\%), which could in part explained the apparent difference in survival.

One explanation of the association between the presence of HPV and the better survival may be the increased sensitivity of HPV-positive tumors to radiotherapy. The strong inverse association between HPV and survival among those who received radiotherapy compared with those who received no radiotherapy has been found (Schwartz et al., 2001). Previous studies have noticed that patients with HPV-positive tumors are less likely to have p53 mutations. The absence of p53 mutations in the tumor cell may allow an enhanced response to radiotherapy (Tachezy et al., 2005; Hoffmann et al., 2005). Hafkamp et al. suggested that radiotherapy may decrease the ability of HPV-E6 and E7 to interact with p53 and pRb which supported the hypothesis above (Hafkamp et al., 2004). However, Ritchie et al. found no statistically significant interaction between HPV status and radiotherapy on survival in oral and oropharyngeal cancers by considering separate effects for surgery and radiotherapy (Ritchie et al., 2003). Moreover, better survival was still found in HPV-positive tumors after adjusting for the presence of a p53 mutation (Campisi et al., 2006). Unfortunately, the present study lacks the information of radiotherapy after operation.

Another explanation has been introduced by the indirect effect of HPV infection on field cancerization which may influence the survival of some HNSCC tumors. It was found that HNSCCs expressing HPV-16 E6 and E7 show substantially lower rates of loss of heterozygosity in chromosomal regions $3 p, 9 p$ and $17 p$ compared to HPV-negative tumors (Braakhuis et al., 2004). One could therefore speculate that HPV-induced HNSCCs may be more univocal, whereas those arising from tobacco originate in several areas due to field cancerization. Statistically speaking, having multiple foci may eventually lead to one focus growing faster and thus becoming the dominating lesion. HPV foci, on the other hand, are fewer and would have a lower chance of producing a "champion" lesion that would quickly take over the epithelium (Schlecht et al., 2005). Gillison et al. proposed that HPV-positive tumors may show less exposure to alcohol and tobacco (Gillison et al., 2000). The loss of the two well-known risk factors may decrease the chance of field cancerization and therefore would increase the survival. As shown in our study, patients with HPV-positive tumors indeed had less exposure to tobacco, but more likely to be alcohol consumers. Furthermore, HPV was shown to have an independent prognostic effect even after adjusting the risk factor of smoking. Similar findings had been made in two studies (Schwartz et al., 2001; Ritchie et al., 2003). In the past decades significant progress has been achieved in the field of HPV vaccine development to reduce the incidence of cervical cancer, which has drawn people's attention to the role of HPV in oral cancer for the biological similarities between the epithelium of the cervix and the oral cavity. Although the effect of HPV on oral cancer remains contentious and the mechanism has yet been unknown, our study did find that HPV was an independent prognostic factor that predicted better survival in OSCC, the dominant subset of oral cancer. This finding raises the prognostic value of HPV and furthermore provides an option for improving the prognosis and quality of life for patients with HPV-associated OSCC by HPV vaccine.

\section{Acknowledgements}

We thank Shi-chun Xiong, Tong Xi and Wei-dong Ge for their assistance. This study was supported by grant No. 301273257 from Wuhan University.

\section{References}

Braakhuis BJ, Snijders PJ, Keune WJ, Meijer CJ, RuijterSchippers HJ, Leemans CR, et al. (2004). Genetic patterns in head and neck cancers that contain or lack transcriptionally active human papillomavirus. J Natl Cancer Inst, 96(13): 998-1006.

Brandwein M, Zeitlin J, Nuovo GJ, MacConnell P, Bodian C, Urken M, et al. (1994). HPV detection using "hot start" polymerase chain reaction in patients with oral cancer: a clinicopathological study of 64 patients. Mod Pathol, 7(7): 720-727.

Campisi G, Giovannelli L, Calvino F, Matranga D, Colella G, Di Liberto C, et al. (2006). HPV infection in relation to OSCC histological grading and TNM stage: evaluation by traditional statistics and fuzzy logic 
model. Oral Oncol, 42(6): 638-645.

Dahlstrom KR, Adler-Storthz K, Etzel CJ, Liu Z, Dillon L, El-Naggar AK, et al. (2003). Human papillomavirus type 16 infection and squamous cell carcinoma of the head and neck in never-smokers: a matched pair analysis. Clin Cancer Res, 9(7): 2620-2626.

Decker J, Goldstein JC (1982). Risk factors in head and neck cancer. $N$ Engl J Med, 306(19): 1151-1155.

Gillison ML, Koch WM, Capone RB, Spafford M, Westra $\mathrm{WH}, \mathrm{Wu} \mathrm{L}$, et al. (2000). Evidence for a causal association between human papillomavirus and a subset of head and neck cancers. J Natl Cancer Inst, 92(9): 709-720.

Hafkamp HC, Manni JJ, Speel EJ (2004). Role of human papillomavirus in the development of head and neck squamous cell carcinomas. Acta Otolaryngol, 124(4): 520-526.

Hansson BG, Rosenquist K, Antonsson A, Wennerberg J, Schildt EB, Bladström A, et al. (2005). Strong association between infection with human papillomavirus and oral and oropharyngeal squamous cell carcinoma: a population-based case-control study in southern Sweden. Acta Otolaryngol, 125(12): 1337-1344.

Hoffmann M, Görögh T, Gottschlich S, Lohrey C, Rittgen $\mathrm{W}$, Ambrosch P, et al. (2005). Human papillomaviruses in head and neck cancer: 8 year-survival-analysis of 73 patients. Cancer Lett, 218(2): 199-206.

Kansky AA, Poljak M, Seme K, Kocjan BJ, Gale N, Luzar $\mathrm{B}$, et al. (2003). Human papillomavirus DNA in oral squamous cell carcinomas and normal oral mucosa. Acta Virol, 47(1): 11-16.

Lo Muzio L, Campisi G, Giovannelli L, Ammatuna P, Greco I, Staibano S, et al. (2004). HPV DNA and survivin expression in epithelial oral carcinogenesis: a relationship? Oral Oncol, 40(7): 736-741.

Lombard I, Vincent-Salomon A, Validire P, Zafrani B, de la Rochefordière A, Clough K, et al. (1998). Human papillomavirus genotype as a major determinant of the course of cervical cancer. J Clin Oncol, 16(8): 26132619.

Pintos J, Franco EL, Black MJ, Bergeron J, Arella M (1999). Human papillomavirus and prognoses of patients with cancers of the upper aerodigestive tract. Cancer, 85(9): 1903-1909.

Remmerbach TW, Brinckmann UG, Hemprich A, Chekol
M, Kühndel K, Liebert UG (2004). PCR detection of human papillomavirus of the mucosa: comparison between MY09/11 and $\mathrm{GP}^{+} / 6^{+}$primer sets. J Clin Virol, 30(4): 302-308.

Ritchie JM, Smith EM, Summersgill KF, Hoffman HT, Wang D, Klussmann JP, et al. (2003). Human papillomavirus infection as a prognostic factor in carcinomas of the oral cavity and oropharynx. Int J Cancer, 104(3): 336-344.

Saiki RK, Scharf S, Faloona F, Mullis KB, Horn GT, Erlich HA, et al. (1985). Enzymatic amplification of betaglobin genomic sequences and restriction site analysis for diagnosis of sickle cell anemia. Science, 230(4732): $1350-1354$.

Schlecht NF (2005). Prognostic value of human papillomavirus in the survival of head and neck cancer patients: an overview of the evidence. Oncol Rep, 14(5): 12391247.

Schwartz SR, Yueh B, McDougall JK, Daling JR, Schwartz SM (2001). Human papillomavirus infection and survival in oral squamous cell cancer: a populationbased study. Otolaryngol Head Neck Surg, 125(1): 1- 9.

Sobin LH, Wittekind C (1997). TNM Classification of Malignant Tumors. 5th ed. New York: Wiley-Liss.

Sugiyama M, Bhawal UK, Dohmen T, Ono S, Miyauchi M, Ishikawa T (2003). Detection of human papillomavirus-16 and HPV-18 DNA in normal, dysplastic, and malignant oral epithelium. Oral Surg Oral Med Oral Pathol Oral Radiol Endod, 95(5): 594-600.

Syrjänen K, Syrjänen S, Lamberg M, Pyrhönen S, Nuutinen J (1983). Morphological and immunohistochemical evidence suggesting human papillomavirus (HPV) involvement in oral squamous cell carcinogenesis. Int J Oral Surg, 12(6): 418-424.

Syrjänen S (2005). Human papillomavirus (HPV) in head and neck cancer. J Clin Virol, 32(Suppl 1): S59-66.

Tachezy R, Klozar J, Saláková M, Smith E, Turek L, Betka J, et al. (2005). HPV and other risk factors of oral cavity/oropharyngeal cancer in the Czech Republic. Oral Dis, 11(3): 181-185.

Yang YY, Koh LW, Tsai JH, Tsai CH, Wong EF, Lin SJ, et al. (2004). Involvement of viral and chemical factors with oral cancer in Taiwan. Jpn J Clin Oncol, 34(4): 176-183.

\footnotetext{
${ }^{\mathrm{a}}$ The authors who contributed equally to this work.

*Corresponding author: Ming-wen Fan

Address: School \& Hospital of Stomatology, Wuhan University, Luoyu Road 237, Wuhan 430079, China

Tel: 862787647443 Fax:862787647443_E-mail: fmw@whuss.com
} 